\title{
Protéger des espaces naturels: constat d'une efficience limitée et propositions pour changer de paradigme
}

\author{
Gaëlle Ronsin* \\ Sociologie, Centre Alexandre Koyré, Paris, France
}

\begin{abstract}
Résumé - La formulation des risques associés à l'érosion de la biodiversité prend de l'ampleur dans les champs politique et scientifique. Plusieurs évènements nationaux et internationaux en 2020 et 2021 mettent en exergue l'importance de cet enjeu via la protection de la biodiversité et des territoires dits naturels. Pourtant, les stratégies nationales ou internationales sont peu convaincantes, d'un point de vue écologique, social, politique ou juridique. Deux colloques organisés à l'automne 2020, l'un par l'Association pour l'histoire de la protection de la nature et de l'environnement, l'autre par la Fondation pour la recherche sur la biodiversité, ont permis de dresser un panorama des recherches et des problématiques actuelles, ainsi que de discuter des enjeux politiques, nationaux et internationaux, de la protection de la nature de façon interdisciplinaire.
\end{abstract}

Mots-clés : recherche / environnement / espaces protégés / biodiversité / gestion

\begin{abstract}
Protected areas: limited efficiency and proposals for a paradigmal shift. Formulation of the risks associated with the global erosion of biodiversity is gaining in importance in political and scientific fields. Several national and international events in 2020 and 2021 highlight the importance of this issue through the conservation of biodiversity and of so-called natural territories. However, the national or international strategies are not fully convincing from an ecological, social, political or legal point of view. Two colloquia organized in the autumn of 2020, one by the Association for the History of Nature and Environmental Protection, the other by the Foundation for Research on Biodiversity, provided an overview of current research and issues, as well as an opportunity to discuss national and international political stakes of nature conservation, with an interdisciplinary focus.
\end{abstract}

Keywords: research / environment / protected areas / biodiversity / management

\section{Introduction}

L'année 2020 devait être marquée par deux rendezvous internationaux consacrés à la protection de la biodiversité. Le Congrès mondial de la nature - une manifestation d'experts organisée par l'Union internationale pour la conservation de la nature (UICN) tous les quatre ans - est présenté comme une étape cruciale pour appuyer les négociations de la $15^{\mathrm{e}}$ conférence des parties (COP 15) de la Convention sur la diversité biologique (CDB). La révision de la stratégie mondiale sur la protection de la biodiversité devrait être discutée à cette occasion, dans le contexte de la publication récente du rapport Global Biodiversity Outlook 5 (CDB, 2020) qui

\footnotetext{
*Auteur correspondant : gaelle.ronsin@gmail.com
}

constate l'échec de cette stratégie : aucun des 20 objectifs d'Aichi adoptés en 2010 n'a été entièrement atteint.

Reportés à 2021 en raison de la pandémie mondiale de Covid-19, les enjeux liés à l'érosion de la biodiversité ont tout de même connu une actualité scientifique importante en France en 2020. Un premier atlas des espaces protégés français, sous-titré Des territoires en partage, a été publié (Laslaz et al., 2020) et présente un état des lieux problématisé (en matière d'acceptation, de conflits, de choix politiques ou de détermination des limites) de ces territoires d'action publique ${ }^{1}$. Deux colloques se sont également déroulés à l'automne 2020 sur ces sujets. Le colloque «De la réserve intégrale à la nature ordinaire. Les figures changeantes de la protection de la nature $\left(\mathrm{XIX}^{\mathrm{e}}-\mathrm{XXI}^{\mathrm{e}}\right.$ siècle) $)$ a été organisé les 29 et

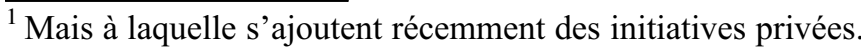


30 septembre par l'Association pour l'histoire de la protection de la nature et de l'environnement (AHPNE) en lien avec les archives nationales et le comité d'histoire $\mathrm{du}$ ministère de la Transition écologique et solidaire ${ }^{2}$. Avec des communications ancrées en histoire, droit, géographie, écologie et philosophie, il a eu pour ambition d'aborder les trajectoires historiques de la conservation de la nature en France métropolitaine et leur coexistence dans un panorama aujourd'hui diversifié de politiques, d'outils et de principes de protection ${ }^{3}$. Le 3 novembre se tenait également la journée d'études annuelle de la Fondation pour la recherche sur la biodiversité (FRB), intitulée "Les aires protégées peuvent-elles sauver la biodiversité au $\mathrm{XXI}^{\mathrm{e}}$ siècle ?» et consacrée à la situation, à l'évaluation et à la discussion de ce principal outil de conservation des espaces naturels en France et dans le monde ${ }^{4}$. Ces deux évènements se sont donc penchés sur la fabrique et l'évaluation de ces territoires protégés, dans le contexte de la révision de la Stratégie nationale pour la biodiversité entreprise en France depuis cette même année.

Ces manifestations scientifiques s'inscrivaient donc dans un contexte politique particulier. Les intervenants étaient d'ailleurs, dans leur majorité, impliqués directement dans le champ des politiques de la nature, par leurs engagements dans des dispositifs (comité de pilotage, conseil scientifique ou autre) ou, pour les intervenants professionnels, par leur travail quotidien dans des structures visant la protection de la biodiversité, dans toute leur pluralité (espaces protégés, conservatoire d'espaces naturels, ministère en charge de l'écologie, Office français pour la biodiversité, etc.).

Ces deux colloques entendaient proposer un retour réflexif sur les pratiques de protection de la nature afin de les adapter aux enjeux environnementaux actuels. Deux interrogations ont traversé les débats: premièrement, la persistance vue comme nécessaire, malgré les critiques, d'un modèle de préservation d'espaces naturels (princi-

\footnotetext{
${ }^{2}$ D'autres partenaires se sont joints : l'Agence française de la biodiversité, le comité français de l'UICN, la fondation François Sommer, le Groupe d'histoire des zones humides, l'Université de Caen-Normandie, l'Université Paris Lumières, la Société française du droit de l'environnement, la Société italienne d'histoire de la faune et la Société nationale de protection de la nature.

${ }^{3}$ Le programme, le livret des interventions et les enregistrements vidéo de ce colloque sont disponibles à cette adresse : http://www.cgedd.developpement-durable.gouv.fr/retour-ducolloque-de-la-reserve-integrale-a-la-a2902.html.

${ }^{4}$ Le programme, les actes de cette journée et les enregistrements vidéo sont disponibles à cette adresse: https://www. fondationbiodiversite.fr/evenement/journees-frb-2020-lesaires-protegees-peuvent-elles-sauver-la-biodiversite-au-xxiesiecle/.
}

palement remarquables) et, deuxièmement, la mise en avant de nouveaux concepts et modalités d'action pour repenser des outils de protection.

\section{Des raisons plurielles pour justifier le développement des espaces naturels protégés}

Comme l'a rappelé en ouverture de la journée de la FRB, son président Jean-François Sylvain, l'anthropisation croissante de la planète s'est traduite par un effondrement des populations d'êtres vivants qui ne cesse de s'amplifier. Le terme de «biodiversité », pour la philosophe Virginie Maris (CNRS) lors du colloque de l'AHPNE, permet justement de "capturer ce moment d'urgence», celui de la sixième crise d'extinction majeure qui engendre un régime de raréfaction des espèces et des milieux naturels. La disparition de la diversité biologique, la fragmentation des espaces naturels ou les ruptures dans les frontières entre sauvage et domestique (Arpin, 2020) conduisent à un accroissement des risques (sanitaires, alimentaires, culturels, environnementaux), qui atteignent un seuil inégalé, comme la pandémie de Covid-19 l'a prouvé depuis 2019 (Hermesse et al., 2020). Face à ces menaces, les aires protégées sont considérées dans le monde occidental, à partir de la fin du $\mathrm{XIX}^{\mathrm{e}}$ siècle, comme «un outil incontournable» pour sauvegarder et protéger la biodiversité et assurer le maintien des services essentiels au devenir des populations humaines.

Depuis le sommet de la Terre de Rio de Janeiro en 1992, la surface des aires protégées a doublé dans le monde. La carte de la base de données mondiale sur les aires protégées, gérée par le World Conservation Monitoring Centre du programme des Nations unies pour l'environnement et l'UICN, annonce la protection de $15,03 \%$ de la surface terrestre et de $7,56 \%$ des superficies marines, selon des réglementations et degrés divers. En France, les espaces protégés couvrent environ $26 \%$ du territoire français (métropolitain et ultramarin). Cette expansion quantitative est avant tout une réponse, comme l'a rappelé à la journée de la FRB, Yann Werhling, ambassadeur français à l'environnement, aux exigences des conventions internationales - notamment à l'objectif 11 d'Aichi, le seul ayant approché une réussite, qui obligeait les États signataires à protéger $17 \%$ de leur territoire terrestre et de leurs eaux intérieures ainsi que $10 \%$ de leurs zones marines et côtières d'ici 2020. L'intervenant a expliqué ainsi que les négociations liées à la révision de la $\mathrm{CDB}$ ont fait apparaître de nouveaux objectifs de protection territoriale avec des cibles encore plus ambitieuses : $30 \%$ de protection, dont $10 \%$ sous protection forte. Mais les dernières versions du texte auraient vu l'objectif de $10 \%$ disparaître. Le blocage viendrait surtout des inquiétudes 
des grands pays pêcheurs, telle la Chine, qui craignent l'atteinte portée à des activités économiques.

Ces engagements entraînent une "course au pourcentage » d'aires protégées (Aubertin et Rodary, 2008), parfois peu intelligible sur les territoires et qui remettent en cause leur acceptabilité. Isabelle Arpin (INRAE) a expliqué qu'en raison d'échecs lors de la création de nouvelles aires protégées et de la récurrence de certains conflits, la participation renforcée des populations a alors été plébiscitée dans les aires protégées. Mais, accroître technocratiquement la participation n'est pas nécessairement la meilleure option et peut entraîner des détournements. Lionel Laslaz (Université Savoie Mont Blanc), dans une intervention sur l'acceptation sociale des parcs nationaux a relaté comment les chartes, pensées pour favoriser la participation, sont devenues des objets hautement conflictuels.

Il a été également rappelé lors de ces colloques que les associations de peuples autochtones s'opposent particulièrement à cet objectif des $30 \%$ et aux dérives qu'il peut entraîner. L'instrumentalisation des aires protégées en vue de la perpétuation ou du renforcement de politiques néocoloniales, processus que l'historien Guillaume Blanc résume sous la formule du «colonialisme vert» (Blanc, 2020), est l'une des principales conséquences négatives de cette approche chiffrée.

Ces tendances à l'accroissement doivent être rapprochées, a rappelé J.-F. Sylvain, des difficultés rencontrées par ces dispositifs, d'abord en termes de moyens : moins d'un quart des aires protégées dispose de ressources suffisantes en personnel et budget. Harold Levrel (AgroParisTech) a montré à la journée de la FRB comment les dépenses de l'État français ont augmenté dans ce secteur jusqu'en 2012, pour ensuite se stabiliser sans qu'il y ait eu de prise de relais par d'autres acteurs. Ce déficit est assez généralisé : au Brésil, les aires protégées présentent un déficit de 24 à $90 \%$ par rapport au budget nécessaire, le plus fort déficit étant pour la forêt amazonienne. En zones Natura 2000, les budgets alloués sont d'environ 150 millions d'euros par an alors que les besoins seraient de 370 millions par an. Les logiques de protection sont ainsi touchées : $4 \%$ des espèces d'amphibiens sont représentées dans des aires protégées dont les moyens sont en adéquation avec les objectifs, alors que $41 \%$ de ces espèces sont menacées (source UICN).

Deuxièmement, on assiste également à une tendance forte à la réduction des surfaces et au déclassement des aires protégées. Ce mouvement, peu présent en Europe, se renforce ailleurs dans le monde, principalement depuis 2000 , où 73 pays ont agi dans ce sens en retirant ou affaiblissant des protections sur plus de $500000 \mathrm{~km}^{2}$. La majorité de ces modifications de statut a pour origine l'extraction industrielle de ressources. Avec les changements d'usage de sols et les transformations climatiques rapides dans les pays du Sud, les aires protégées deviennent aussi de plus en plus des surfaces à exploiter pour les populations.

Malgré les critiques que l'on peut leur adresser, François Sarrazin (Sorbonne Université) a invité à réfléchir aux risques à «ne pas développer les aires protégées ». Le maintien dans les aires protégées de communautés biologiques fonctionnelles ayant une diversité spécifique élevée permettrait, par exemple, d'éviter, grâce à leur rôle tampon, l'émergence de pathogènes majeurs susceptibles d'être à l'origine de zoonoses, comme le met en évidence une étude comparative entre districts plus ou moins anthropisés menée au Costa Rica par l'équipe de Brett R. Bayles (Bayles et al., 2020, cité par Hélène Soubelet, directrice de la FRB). F. Sarrazin a appelé ainsi à renverser la charge de la preuve, à " passer de "pourquoi protéger" à "pourquoi détruire" » en questionnant les choix individuels et collectifs. Pour lui, le saisissement de cet outil et la protection des «cathédrales de la nature» peuvent entraîner d'importantes innovations éthiques, sociales, politiques, économiques, juridiques ou foncières.

Lors des deux colloques, des interventions ont rappelé que les raisons qui conduisent à réserver certaines portions du territoire à la protection de la nature ont évolué dans le temps. Pour Cécile Albert (journée de la FRB), écologue au CNRS, quatre critères ont pu être mis en avant pour choisir les territoires à préserver : la représentativité (la globalité), l'exhaustivité (la richesse), l'irremplaçabilité (la rareté) et enfin la complémentarité (la variété en réseau). La chercheuse a évoqué l'existence d'outils de priorisation spatiale pour intégrer des critères socioéconomiques à ces approches. En effet, des raisons d'un autre ordre guident aussi ces choix politiques. La première réserve de l'histoire, créée en 1853 à Fontainebleau, avait, a expliqué V. Maris, un objectif esthétique. Il s'agissait de protéger un patrimoine et de déroger aux règles forestières habituelles. Dès le départ, il s'agissait donc d'une «protection contre », en rupture avec un mouvement d'anthropisation. Le géographe Bernard Sajaloli (Université d'Orléans) a mené une étude sur la représentativité des zones humides dans les réserves. Sa communication, lors du colloque de l'AHPNE, a établi que $70 \%$ des réserves naturelles (régionales ou nationales) possèdent dans leurs limites aujourd'hui des zones humides d'importance. Pour lui, ces chiffres et leurs évolutions démontrent qu'il existe des «phénomènes de mode» dans la protection de certains milieux naturels par rapport à d'autres. Ainsi, les zones humides, peu considérées scientifiquement au $\mathrm{XIX}^{\mathrm{e}}$ siècle, connaissent un engouement autour des années 1990 grâce à l'attention portée sur ces écosystèmes par des chercheurs. Cette focalisation traduit également un phénomène politique. Dans la région Hauts-de-France, l'arrivée d'une présidence 
écologiste voit apparaître des projets territoriaux nouveaux et la protection de nombreuses zones humides. Chaque type de zone humide (les tourbières ou les mangroves, par exemple) connaît également des «moments» propices à sa protection. Il existe donc bien une variation dans le temps des représentations de la nature à protéger pour les scientifiques et protecteurs, liées «aux regards, connaissances et préoccupations de leur temps». Depuis 2010, la priorité stratégique est donnée au développement des aires marines protégées avec, par exemple la création du parc naturel marin de la mer de Corail en Nouvelle-Calédonie. B. Sajaloli a donc appelé à une «humilité nécessaire des politiques et des actions de protection de la nature pour considérer leur pertinence, qui évoluera forcément avec le temps ».

Les communicants ont conclu qu'aujourd'hui les justifications à créer des aires protégées s'empilent. Entre la protection d'une wilderness, de la biodiversité ou de services écosystémiques (écologiques, culturels ou récréatifs), les aires protégées sont perçues désormais comme un outil privilégié d'atténuation ou d'adaptation face aux changements globaux accélérés (Melillo et al., 2016) mais aussi comme des protections, ainsi que l'a rappelé V. Maris, contre l'extension du régime capitaloindustriel d'exploitation des ressources.

\section{Penser des paradigmes dynamiques pour améliorer les aires protégées}

«Les aires protégées ne suffiront probablement pas à sauver la biodiversité au XXI ${ }^{\mathrm{e}}$ siècle » a martelé François Gauthiez, directeur de l'appui aux stratégies pour la biodiversité à l'Office français de la biodiversité. L'efficacité de cet outil mais aussi ses limites et les évolutions à y apporter, théoriquement et empiriquement, ont été largement discutées lors de ces deux colloques.

Peu d'études mesurent l'efficacité directe des aires protégées sur la biodiversité, même si des effets positifs sont relevés, telles que l'abondance et la richesse en espèces menacées. Leur localisation dans des territoires déjà peu anthropisés (par exemple dans les zones montagnardes en France métropolitaine) doit toutefois amener à relativiser ce constat. Joachim Claudet (CNRS) a développé une classification des aires marines protégées (AMP) en fonction de leur niveau de protection réel ainsi que de la qualité de la gestion. Pour beaucoup d'AMP, notamment en Méditerranée, la différence entre intérieur et extérieur est peu signifiante. Finalement, de façon peu surprenante, ce sont les aires les mieux gérées qui sont les plus efficaces, quel que soit le niveau de protection.

L'efficacité des aires protégées peut être aussi évaluée, selon Ana Rodrigues (CNRS), en appréhendant les pressions anthropiques. Par rapport à des sites similaires non protégés, les aires protégées terrestres connaissent des taux de déforestation plus faibles, ont des niveaux stables de lumière nocturne artificielle, et sont l'objet d'une augmentation de l'empreinte humaine plus lente.

Pour Pierre Chassé (AgroParisTech), lors du colloque de l'AHPNE, la conservation de la biodiversité ne doit pas être un problème appréhendé de façon uniquement scientifique et technique, même si elle est habituellement traitée de cette manière, comme le prouvent les deux approches précédentes. La réforme récente du Conseil national de protection de la nature, qu'il étudie, s'inscrit pourtant dans cette tendance en privilégiant une composition experte et rationnelle au détriment de l'intervention d'acteurs associatifs ou de représentants d'intérêts plus larges.

Le principe des aires protégées implique donc toujours une vision relativement statique de la nature et de ses acteurs. Pourtant, depuis longtemps, les scientifiques plaident pour une vision plus dynamique de la conservation, résumée sous le mot d'ordre «protéger des processus plutôt que des états ». Au-delà d'un seul territoire protégé, les interventions ont montré qu'il s'agit désormais d'appréhender les enjeux de conservation à des échelles géographiques, sociales et temporelles plus vastes et de façon plus systémique. Cette approche a été ainsi saisie de trois façons dans ces colloques : temporellement, spatialement et sectoriellement.

Premièrement, C. Albert a invité à mieux appréhender les aspects temporels. En raison des changements climatiques, les aires protégées des biomes tempérés et de haute latitude nord affronteront des conditions climatiques nouvelles aux échelles locales, régionales et globales d'ici la fin du siècle, ce qui sous-entend des perturbations significatives de leur fonctionnement écologique (migrations d'espèces hors des aires protégées, entrée d'espèces exotiques envahissantes). Sur le long terme, il est donc nécessaire de préserver les flux de gènes et les interactions biotiques pour permettre l'adaptation des organismes et leur évolution. Mais comme l'a expliqué V.Maris, les prédictions sont difficiles et les trajectoires seront de très long terme. Il s'agit alors, selon elle, de renverser la logique: ne pas chercher à prédire à chaque instant ce qui sera présent et où, mais s'évertuer à réduire nos impacts et atténuer la pression, sans réduire l'ambition des projets de protection.

Deuxièmement, à l'échelle spatiale, la conservation de la biodiversité doit relever deux objectifs : assurer le maintien de dynamiques écologiques locales (dynamiques de populations, processus démographiques, besoin de populations locales assez grandes), mais également assurer les déplacements (annuels et saisonniers) des 
individus pour leurs besoins (reproduction, colonisation de nouveaux habitats, interactions biotiques, flux spatiaux...). C'est l'objet des trames vertes et bleues et des politiques qui visent à protéger la biodiversité dite "plus ordinaire» en maintenant des connectivités entre habitats. Cette biodiversité ordinaire, peu discutée dans la journée d'études de la FRB, a fait l'objet d'interventions plus amples lors du colloque de l'AHPNE, notamment lors de la conférence introductive de Raphaël Larrère qui a retracé la genèse de cette notion, nécessaire selon lui pour sortir de l'unique protection du «remarquable» et d'une nature «remarquée».

La concrétisation de cette protection est complexe. La « connectivité écologique» est l'un des principes mis en avant et censé appuyer cette idée. J.-F. Sylvain a plaidé pour assurer un fort degré de connectivité entre les aires protégées. En effet, seul $9,7 \%$ du réseau des aires protégées terrestres est considéré comme «structurellement connecté » malgré les fortes incitations scientifiques en la matière. La façon de promouvoir cette connectivité a été discutée à partir par exemple d'une étude sur la région de Montréal (vallée du Saint-Laurent) où les chercheurs ont identifié un réseau d'habitats forestiers qui permet à la fois le maintien des populations localement, mais qui autorise également les connexions entre les massifs forestiers du Sud-Est et du Nord-Est de l'agglomération, en prenant en compte les besoins en habitat et la capacité de dispersion de plusieurs espèces de vertébrés.

La protection de zones périphériques peut s'appuyer sur des acteurs et dynamiques au-delà des gestionnaires publics. Ainsi, pendant la journée de la FRB, Vincent Leblan (IRD), à partir de ses recherches sur les parcs en Guinée, en Guinée-Bissau et au Mali, a montré en quoi des pratiques agricoles et sociales à proximité des parcs peuvent être favorables à ces zones de conservation: «L'agriculture sur brûlis crée des jachères qui profitent par exemple aux chimpanzés. De jachères en jachères, les animaux sont plus nombreux». Au Mali, le système gérontocratique contraint les jeunes à quitter leurs villages pour s'installer. "Les meilleures terres sont aux plus âgés, alors les jeunes vont en chercher ailleurs, puis ils reviennent au village. Ainsi, des fronts de culture apparaissent, puis sont abandonnés, des manguiers et des baobabs en profitent, et cela est bénéfique aux animaux ».

Afin de donner vie à une approche plus évolutive de la conservation, une proposition de créer un réseau « d'aires marines dynamiques » dans l'océan Austral est portée par Yan Ropert-Coudert (CNRS). Dans une étude publiée récemment dans la revue Nature (Ropert-Coudert et al., 2020), les chercheurs proposent ainsi d'identifier les zones à protéger de l'océan Austral en fonction de la localisation de prédateurs marins. Ce principe a été théoriquement développé à partir de la construction d'une base de données portant sur le suivi de
4000 individus de 17 espèces différentes (phoques, éléphants de mer, baleines) qui a permis d'identifier des «zones d'importance écologique», avec une forte abondance de plusieurs espèces (prédateurs et autres), qui recoupent partiellement des aires marines protégées existantes. D'après les projections du Groupe d'experts intergouvernemental sur l'évolution du climat (Giec), ces zones ne se recouvriront plus d'ici 2100 . Ce constat a donc incité les chercheurs à proposer un nouveau paradigme pour la conservation de ces zones. Les aires protégées pourront alors suivre les évolutions climatiques. Politiquement, l'intégration de ces approches semble longue et complexe en raison des enjeux économiques, géopolitiques et de gouvernance qui traversent cette zone internationale. Pour l'instant, seules deux de ces aires marines protégées ont été acceptées par la Commission pour la conservation de la faune et la flore marines de l'Antarctique, et seulement au niveau de la zone Antarctique stricte (en dessous de $60^{\circ}$ de latitude).

Troisièmement, les débats scientifiques et politiques retracés dans ces colloques montrent que des acteurs de la conservation cherchent à intégrer la biodiversité dans des politiques sectorielles (agriculture, énergie, transports) et territoriales (urbanisme, planification, financement, contractualisation) et à s'intéresser à ses acteurs. Plusieurs leviers d'action ont été discutés, dont, en premier lieu, la révision des instruments d'action publique. Les études d'impact sont, par exemple, un instrument répandu, a expliqué la juriste Marthe Lucas (Université d'Avignon) lors du colloque de l'AHPNE, mais qui est encore trop peu mobilisé à la hauteur de ses possibilités. Ainsi, les modalités de compensation demandées par les juges administratifs lors de l'application de la séquence "Éviter, réduire, compenser » sont encore bien trop faibles, à l'exception des zones humides, et ne permettent pas d'aboutir à l'objectif exigé de «zéro perte nette de biodiversité».

Certaines structures tentent d'inventer d'autres paradigmes pour orienter leurs actions et dépasser les instruments d'action publique. Les conservatoires d'espaces naturels, représentés par Pascal Marty, Claudie Houssard, Sonia Bertrand, mettent ainsi en avant la notion «d'intendance du territoire» pour « rénover les outils de la régulation publique $»$. Ce concept vise à se détacher de l'approche réglementaire des politiques publiques et à contrer une dérive néolibérale croissante dans le champ de la conservation, en proposant une logique plus «bottom-up» des modalités de gestion. Concrètement, il s'agirait d'élargir les acteurs impliqués dans la conservation de territoires naturels, notamment en proposant une gouvernance partagée, appuyée à la fois sur la propriété foncière et les usages réels de l'espace. À l'inverse, des démarches promouvant uniquement la propriété privée, comme seule garante d'une protection, sont défendues par des associations et des scientifiques, 
comme l'a présenté Madline Rubin, directrice de l'Association pour la protection des animaux sauvages (Aspas) à la journée de la FRB. Dès le début du $\mathrm{XX}^{\mathrm{e}}$ siècle, des réserves ornithologiques ou zoologiques sont créées sur des propriétés foncières acquises ou louées par des associations de naturalistes. En Europe, depuis les années 2000 (Locquet et Héritier, 2020), des ONG d'un autre type (Aspas, Mountain Wilderness, Rewilding Europe) promeuvent le repli des usages anthropiques (chasse, agriculture), la libre évolution et le réensauvagement des milieux par la création de réserves privées (appelées «réserves de vie sauvage» par l'Aspas) pour se démarquer des situations de multifonctionnalité existant dans les espaces protégés par la puissance publique. Ces approches, soutenues financièrement par des donateurs, donnent lieu à des débats et tensions parfois vives localement et interrogent le dialogue territorial et l'équité des usages que peuvent ou non catalyser des aires protégées.

Au-delà de la gouvernance, des initiatives territoriales réunissant de façon plus ou moins serrée gestionnaires, scientifiques et acteurs, cherchent à déplacer la conservation sur d'autres terrains, par exemple en ville ou dans des quartiers, comme l'ont relaté l'écologue Sébastien Bonthoux (Insa de Blois) ou la géographe Léa Billen (Université Paris Nanterre) lors du colloque de l'AHPNE. De nouveaux environnements sont aussi investigués, comme le montre la construction récente, retracée par Johan Milian (Université Paris 8), de politiques de conservation dédiées à l'environnement nocturne. Certaines espèces, certains paysages et processus écologiques spécifiquement liés à la nuit sont dégradés par la pollution lumineuse. Des labels (comme les réserves internationales de ciel étoilé) ou des dispositifs (la trame noire) voient le jour et traduisent des intentions et mobilisations d'acteurs plutôt que des réponses à cette pollution (Laslaz et al., 2020).

Enfin, des courants de pensée discutent également la forme d'interventionnisme qui s'est développée dans la gestion des espaces protégés et, plus largement, de la nature. Le paradigme du " pilotage de la biodiversité » est remis en cause comme «une dérive», a relaté le géographe Régis Barraud (Université de Poitiers), auquel les structures préfèrent désormais le concept de «libre évolution" dans le but de desserrer des pratiques de gestion. Mais sans que cela entraîne forcément des modifications fortes dans les pratiques des gestionnaires.

Finalement, ces paradigmes de gestion des espaces naturels, anciens ou plus récents, constituent des modes d'institution sociale des rapports homme-nature. Leur caractère normatif transparaît encore fortement, malgré la volonté de faire évoluer leurs modalités.

\section{Conclusion}

Les fronts scientifiques sur le sujet de la protection de la nature ou de la biodiversité et ses enjeux juridiques, écologiques, politiques, spatiaux ou philosophiques sont nombreux. Face aux menaces croissantes, les apports théoriques récents s'arriment de façon pragmatique aux agendas politiques, qu'ils soient régionaux, nationaux ou internationaux. Des leviers d'action (instrumentaux ou organisationnels), leurs paradigmes, leurs modalités opérationnelles mais aussi leur efficacité sont désormais scrutés scientifiquement, comme le montrent les deux colloques dont cet article propose le compte rendu. Les aires protégées apparaissent toujours dans les discours comme un outil important, à la fois quantitativement mais aussi pour leur caractère performatif. En les concevant de façon plus dynamique, ces espaces pourraient permettre d'expérimenter de nouveaux rapports à la nature, extraits du système capitaliste. La concrétisation de ces transformations est toutefois encore timide. Et, malgré toutes les critiques qui leur sont adressées, il ne semble pas envisageable pour les communicants de supprimer des aires protégées dont les effets escomptés, écologiques et sociaux, n'ont pas été atteints. Au regard des constats dressés et pour envisager le futur des espaces protégés, ne serait-il pas temps, comme le proposent Laslaz et al. (2020) et à rebours des conventions internationales, de cesser toute extension surfacique et de viser plutôt la préservation et l'efficience des aires déjà protégées?

\section{Références}

Arpin I., 2020. Une vie sauvage sans frontières ? Le cas de la contamination des bouquetins du Bargy, Zilsel, 7, 2, 179198, https://doi.org/10.3917/zil.007.0179.

Aubertin C., Rodary E. (Eds), 2008. Aires protégées, espaces durables ?, Montpellier, IRD Éditions, https://doi.org/ 10.4000/books.irdeditions.5655.

Bayles B.R., Rusk A., Christofferson R., Agar G., Pineda M. A., Chen B., Dagy K., Kelly E., Hummel T., Kuwada K., 2020. Spatiotemporal dynamics of vector-borne disease risk across human land-use gradients: examining the role of agriculture, indigenous territories, and protected areas in Costa Rica, The Lancet Global Health, 8, S32, https://doi. org/10.1016/S2214-109X(20)30173-X.

Blanc G., 2020. L'invention du colonialisme vert, Paris, Flammarion.

CDB (Convention sur la diversité biologique), 2020. Global Biodiversity Outlook 5, Montréal, CDB, https://www.cbd. int/gbo5.

Hermesse J., Laugrand F., Laurent P.-J., Mazzocchetti J., Servais O., Vuillemenot A.-M., 2020. Masquer le monde. Pensées d'anthropologues sur la pandémie, Paris, L'Harmattan. 
Laslaz L., Cadoret A., Milian J., 2020. Atlas des espaces protégés en France. Des territoires en partage ?, Paris, Publications scientifiques du Muséum national d'histoire naturelle.

Locquet A., Héritier S., 2020. Interrogation autour de la nature et du sauvage à propos de l'établissement de wilderness areas en Europe, Cybergeo : European Journal of Geography, https://doi.org/10.4000/cybergeo.34986.
Melillo J.M., Lu X., Kicklighter D.W., Reilly J.M., Cai Y., Sokolov A.P., 2016. Protected areas' role in climate-change mitigation, Ambio, 45, 2, 133-145, https://doi.org/10.1007/ s13280-015-0693-1.

Ropert-Coudert Y., Van de Putte A.P., Reisinger R.R. et al., 2020. The retrospective analysis of Antarctic tracking data project, Scientific Data, 7, 94, https://doi.org/10.1038/ s41597-020-0406-x.

Citation de l'article: Ronsin G. Protéger des espaces naturels : constat d'une efficience limitée et propositions pour changer de paradigme. Nat. Sci. Soc. 29, 3, 334-340. 